\title{
Fidelity in Quasi-1D Systems as a Probe for Anderson Localization
}

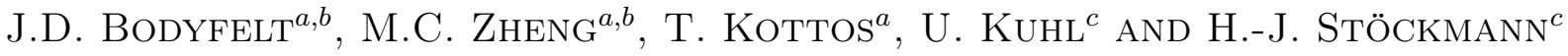 \\ ${ }^{a}$ Department of Physics, Wesleyan University, Middletown, Connecticut 06459, USA \\ ${ }^{b}$ MPI for Dynamics and Self-Organization, Bunsenstraße 10, D-37073 Göttingen, Germany \\ ${ }^{c}$ Fachbereich Physik, Philipps-Universität Marburg, Renthof 5, D-35032 Marburg, Germany
}

\begin{abstract}
We analyze the echo dynamics in quasi-one-dimensional random media to investigate how the transition from localization to delocalization is encoded in its temporal decay properties. Our analysis extends from the standard perturbative regime corresponding to small perturbations (with respect to the mean level spacing) in the echo dynamics, out to the Wigner decay regime. On the theoretical side, our results rely on a banded random matrix modeling, and show in the localized regime under small perturbations a novel decay of the fidelity (Loschmidt echo), differing from the typical Gaussian decay seen within both diffusive and chaotic systems. For larger perturbation strengths, typical Wigner exponential decays are observed. Scattering echo measurements are performed experimentally within a quasi-1D microwave cavity randomly populated with point-like scatterers. Agreements are observed between experiments, numerics, and theoretical predictions.
\end{abstract}

PACS numbers: 42.25.Dd, 72.15.Rn, 03.65.Nk, 03.65.Yz

\section{Introduction}

In both pure physics and real-world engineering, wave propagation captivates us. Such propagation becomes especially interesting in the presence of complex random media, where multiple scattering events produce interesting interference phenomena. One such phenomenon that has drawn considerable attention over the years is Anderson localization, originally proposed in the framework of electron transport. Specifically, 50 years ago in the seminal work of Anderson [1,2], it was found that the classical (diffusive) picture was wrong, which assumed that the electrons perform random walks, yielding survival probability decays $\sim 1 / \sqrt{t}$ and Ohmic conductance of $G \sim 1 / L$, where $L$ is the system length (wire length). Due to wave interferences, a total halt of electronic propagation is found instead - leading to a converging non-zero return probability $\sim 1 / l_{\infty}$ and to an exponential decay of the conductance $G \sim \exp \left(-L / l_{\infty}\right)$, where $l_{\infty}$ is the so-called localization length.

Despite the enormous research efforts by various groups in measuring Anderson localization, it took nearly 40 years to observe localization phenomena beyond any doubt. A decisive step towards this direction was done by optics and microwave experiments, which allow a detailed study of Anderson localization, undisturbed by interactions or other effects which characterize electron propagations $[3,4]$. First experiments showing photon localization [5] had the problem of separating localization from absorption, which can be another source of exponential decay of a propagating electromagnetic wave. A solution to this problem was given by Chabanov et al. in Ref. [6], where they proposed to study the relative size of fluctuations of certain transmission quantities. They found clear evidence of localization in a quasi-one-dimensional (quasi-1D) microwave waveguide with ran- domly distributed dielectric or metallic spheres [6] (see also Fig. 2).

This powerful approach however does not allow a transport view from dynamical perspectives, nor does it make direct contact with the original ideas of Anderson theory, which suggested probing localization by means of small perturbations [7]. Such a possible probe is the Loschmidt echo (LE), or fidelity (for a review, see Ref. [8]). Literature on fidelity is widespread, ranging from atomic physics [9], microwaves [10], elastic waves [11], to quantum information [12] and quantum chaos [13-19]. The fidelity, $F^{\lambda}(t)$, is formally defined [10] as

$$
F^{\lambda}(t) \equiv\left|f^{\lambda}(t)\right|^{2}=\left|\left\langle\psi_{0}\left|\mathrm{e}^{\mathrm{i} H_{0} t / \hbar} \mathrm{e}^{-\mathrm{i} H_{\lambda} t / \hbar}\right| \psi_{0}\right\rangle\right|^{2},
$$

where $f^{\lambda}(t)$ is the fidelity amplitude, $H_{\lambda}=H_{0}+\lambda V$ is a single parameter Hamiltonian family, $H_{0}$ is the unperturbed Hamiltonian, $\lambda V$ is the perturbation of strength $\lambda$ where $\left\langle\left|V_{n m}\right|^{2}\right\rangle=1$, and $\left|\psi_{0}\right\rangle$ is an initial state.

The fidelity in the form of Eq. (1) is hardly accessible experimentally. Therefore, the notion of scattering fidelity was introduced [10] as an experimental alternative to Eq. (1). The scattering fidelity coincides in the case of weak coupling for chaotic systems with the standard definition of fidelity $[10,20]$.

The scattering fidelity amplitude is defined as

$$
\begin{aligned}
& f_{a b}^{\lambda}(t)=\left\langle S_{a b}^{\lambda *}(t) S_{a b}^{0}(t)\right\rangle \\
& \quad / \sqrt{\left\langle\left|S_{a b}^{\lambda}(t)\right|^{2}\right\rangle\left\langle\left|S_{a b}^{0}(t)\right|^{2}\right\rangle},
\end{aligned}
$$

where $S_{a b}(t)$ is one component of the scattering matrix in the time domain, while the indices $a$ and $b$ refer to the antennae involved. The averages are done over disorder realizations. In microwave studies the $S_{a b}$ are directly 
accessible from transmission $(a \neq b)$ or reflection $(a=b)$ measurements in the frequency domain. The corresponding quantities in the time domain are then obtained by Fourier transforms. The superindices $\lambda, 0$ indicate scattering matrix elements corresponding to the perturbed and unperturbed system, respectively. It is important to point out that the denominator in Eq. (2) renormalizes the fidelity decay due to absorption, thus allowing us to trace out localization phenomena [10]. This idea addresses absorption in a similar fashion as Ref. [6].

The structure of the paper is as follows: in the next section we discuss the experimental setup and present statistical measures to quantify (in terms of localization) the various frequency regimes. In the third section, we discuss the random matrix theory (RMT) modeling used to describe diffusive and localized quasi-one-dimensional samples. The temporal behavior of the fidelity decay in the diffusive and localized regime for various perturbation strengths is derived in the fourth section. In the fifth section, we compare our experimental results for the scattering fidelity with the theoretical findings. Our conclusions are finally given within the last section. Part of our results have been published in a recent short paper [21].

\section{Experimental setup and characterization of the sample}

Figure 1 is a photograph of the microwave cavity that was used in our experiments for the scattering fidelity. It is a brass rectangular waveguide with brass bars at two ends closing the waveguide, with dimensions of $8 \mathrm{~mm}$ height, $10 \mathrm{~cm}$ width, and $100 \mathrm{~cm}$ long. One of the short wall-lengths is allowed to move, and a shift in the wall acts as a global perturbation. The cavity is randomly filled with 186 brass cylinders with radius $5 \mathrm{~mm}$. Microwave TE modes excited in the cavity are transverse. The wavelengths are such that the cylinders can be viewed as point scatterers. In the studied frequency range, only $\mathrm{TE}_{n, 0}$ modes can be excited, so the cavity can be considered as quasi-one-dimensional. Two microwave antennae are coupled into the cavity, one close to the perturbing short wall and the other deep within the scattering bulk. The antennae are connected to an Agilent 8720ES VNA, which sends out microwaves in the frequency range of $3-12 \mathrm{GHz}$ at a $100 \mathrm{kHz}$ resolution. The VNA then measures the $S$-matrix elements $S_{a b}(E)$ which are recorded. The perturbation is applied via a position shift of the moveable wall, in increments of $\delta w=0.2 \mathrm{~mm}$, up to a maximum wall shift of $w=18.0 \mathrm{~mm}$. The perturbed $S$-matrix elements are also recorded, and for a given perturbation the scattering fidelity is calculated using Eq. (2). An ensemble average over 15 different realizations of scatterer positions was then performed.

In order to address localization in absorptive systems, the relative size of transmissive fluctuations was used in Ref. [6] to find signatures of localization within a quasi-1D microwave waveguide with randomly distributed dielectric or metallic spheres. The relative size

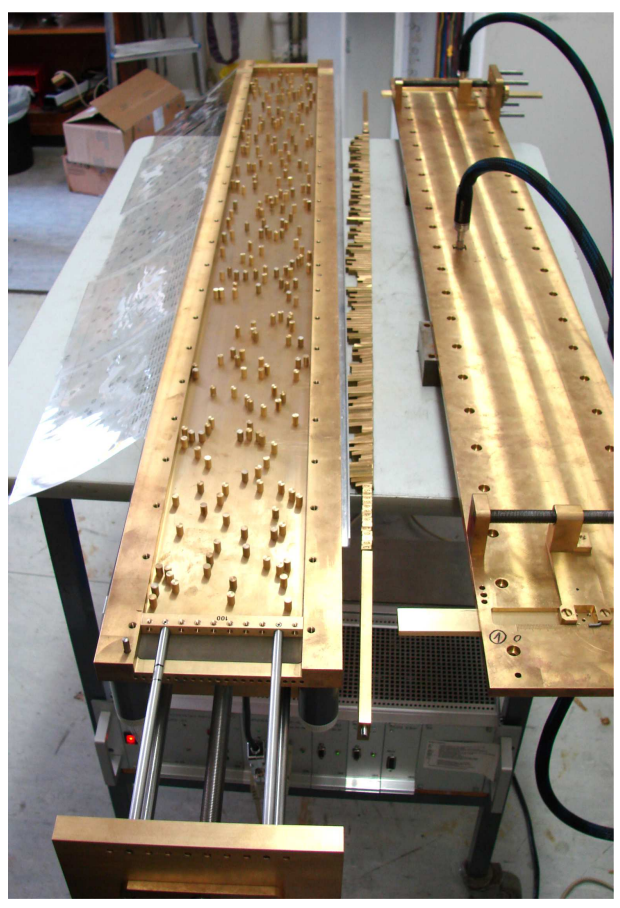

Fig. 1. Photograph of the experimental microwave cavity filled with random scatterers. The top plate of the cavity can be seen at the right side, rotated by $180^{\circ}$. The point scatterers can be seen as small brass cylinders, randomly arranged inside the cavity. The bottom short wall is moveable and acts as a global perturbation.

of the transmissive fluctuations is captured by the variance $\sigma_{\tilde{T}}^{2}$ of the mean normalized transmission intensity [6] defined as

$$
\tilde{T}=\left|S_{21}\right|^{2} /\left\langle\left|S_{21}\right|^{2}\right\rangle \text {. }
$$

Since our experiment does not probe the total transmission but just one component of the scattering matrix, we expect localization whenever $\sigma_{\tilde{T}}^{2}$ exceeds the critical value of $7 / 3[6]$. We find (see Fig. 2) that this condition is satisfied approximately in the frequency window from 5.5 to $9.0 \mathrm{GHz}$. Above $9 \mathrm{GHz}$ the waveguide modes are delocalized, while below $5.5 \mathrm{GHz}$ the values of the variances are error prone, as $\left|S_{21}\right|^{2}$ decays below the noise level of the vector network analyzer $\left(\left|S_{21}\right|^{2}<10^{-6}\right)$. In the delocalized regime random matrix theory predictions are applicable [22], yielding a value of

$$
\sigma_{\tilde{T}}^{2} \approx \frac{\left(2 N_{\mathrm{c}}+1\right)^{2}}{N\left(2 N_{\mathrm{c}}+3\right)}-1,
$$

where $N_{\mathrm{c}}$ is the number of open channels. In the limit $N_{\mathrm{c}} \gg 1$, the variance approaches the value $\sigma_{\tilde{T}}^{2} \approx 1$, in agreement with our experimental data for the high frequency regime. We shall henceforth limit our calculations to two windows; a "localized frequency window" of $6.0-7.5 \mathrm{GHz}\left(N_{\mathrm{c}}=4\right)$ which is highlighted in darker grey within Fig. 2, and a "diffusive frequency window" of $10.5-12.0 \mathrm{GHz}\left(N_{\mathrm{c}}=7\right)$, highlighted in lighter grey. 


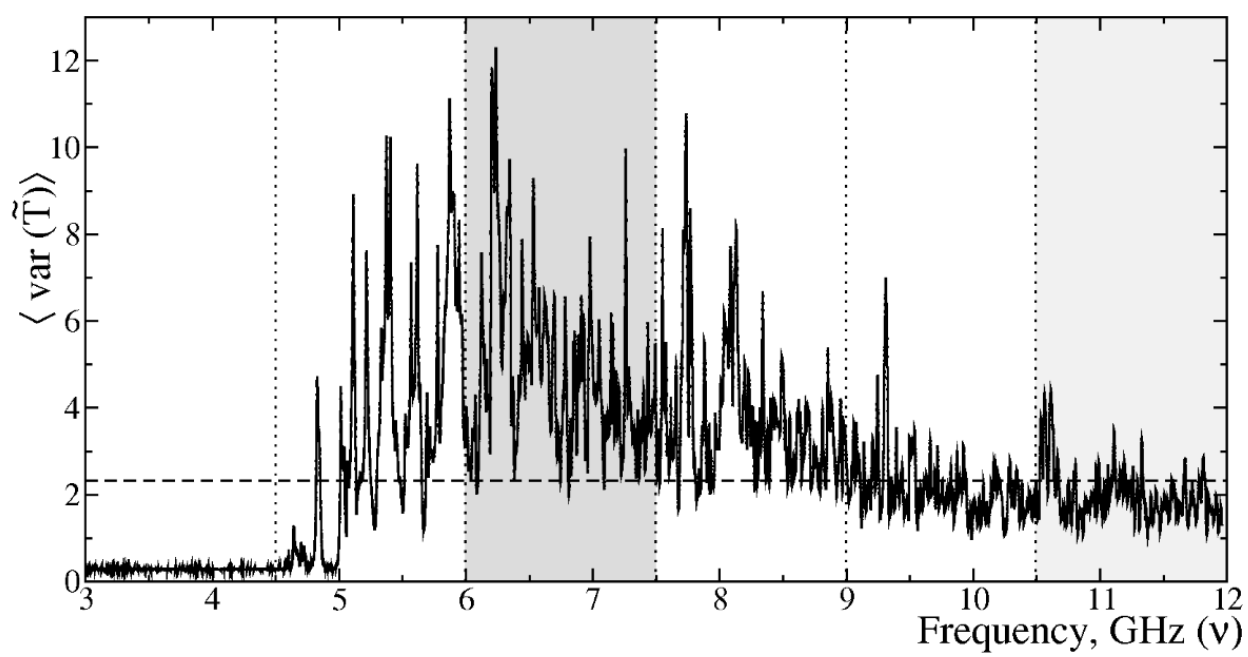

Fig. 2. Average variance of the normalized transmission, $\sigma_{\tilde{T}}^{2}$, as a function of microwave frequency. The horizontal line denotes the theoretical threshold of $7 / 3$. Frequencies with an average variance above this threshold are localized, while those below are diffusive. The vertical dotted lines correspond to frequencies at which a new mode is opened within the system. A localized frequency window is observed for frequencies in 6.0-7.5 GHz (highlighted in darker grey), and a diffusive window is observed for frequencies in 10.5-12.0 GHz (highlighted in lighter grey).

From transmission fluctuations, the normalized transmission should follow Rayleigh-like distributions in the diffusive frequency window, and then broaden out to log-normal distribution behavior in the localized frequency window $[2,23,6]$. The $\log$-normal distribution is defined as

$$
P(\tilde{T}) \sim \exp \left(-\frac{(\ln (\tilde{T})-\langle\ln (\tilde{T})\rangle)^{2}}{2 \sigma_{\tilde{T}}^{2}}\right) .
$$

The experimental distribution is presented (solid line) in Fig. 3, compared with the best fit to Eq. (5) of the peak region (dashed line). An agreement is seen, and therefore we are confident that the microwaves in the frequency regime $6.0-7.5 \mathrm{GHz}$ are localized.

\section{Modeling localization via random matrix theory}

A way to model quasi-1D disordered systems of size $N$ with $b$ propagating channels is provided by random matrix theory modeling. In the early 90's, it was shown [24] that banded random matrices (BRM) can be mapped exactly to the $1 \mathrm{D}$ non-linear $\sigma$ model, which is the appropriate field theoretical model describing quasi-1D disordered systems. Within BRM, elements are drawn from a Gaussian sequence that is subject to a zero mean, but a variance of

$$
\left\langle\left|H_{n m}\right|^{2}\right\rangle= \begin{cases}1+\delta_{n m}, & |n-m| \leq b \\ 0, & |n-m|>b\end{cases}
$$

The limiting case of $b=N / 2$ corresponds to the standard Gaussian orthogonal ensemble (GOE), which describes chaotic systems. The other limit of $b=1$ corresponds to a 1D Anderson tight-binding model, which

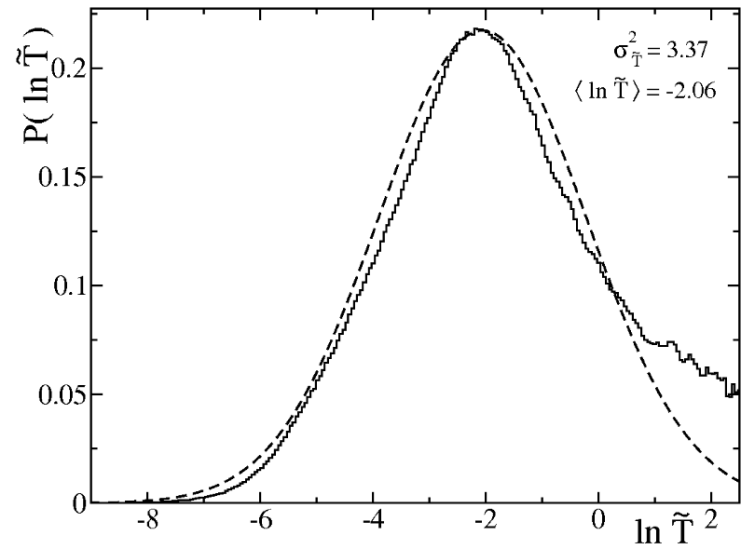

Fig. 3. Distribution of the normalized transmissions within the localized frequency window $(6.0-7.5 \mathrm{GHz})$. The solid line is the actual transmission distribution from the experiment, while the dashed line is a best fit to the log-normal distribution of Eq. (5). The fit parameter $\sigma_{\tilde{T}}^{2}$ is calculated as 3.37 , which is above the threshold value of $7 / 3$.

is known to have exponentially localized wave functions as solutions. For any intermediate value of $b$, the localization properties of the eigenfunctions were investigated both analytically [25] and numerically [26]. It was specifically found that for a finite $N$-dimensional sample with $b<N$, the localization properties of the wave functions scale with the parameter $\Lambda=b^{2} / N$ : for $\Lambda \ll 1$, the BRM eigenvectors show an average exponential decay around some central site (localized), while in the opposite limit of $\Lambda \gg 1$, the BRM eigenvectors are extended through- 
out the sample (diffusive). Past analysis was performed by studying scaling properties of various moments of the eigenvectors, the most popular being the inverse participation number (IPN), defined in its discrete version as

$$
P_{2}=\sum_{n=1}^{N}\left|\psi_{n}\right|^{4},
$$

where $\psi_{n}$ is the eigenfunction amplitude at site $n$. The IPN behaves as $\sim N^{-1}$ for the diffusive case, and $\sim l_{\infty}^{-1}$ for the localized case. In the case of BRM matrices, it was found that the localization length is given by

$$
l_{\infty}(E)=\frac{2}{3} \pi^{2} \rho^{2}(E) b^{2},
$$

where $\rho(E)$ is the density of states, given as

$$
\rho(E)=\left\{\begin{array}{cl}
\frac{1}{4 \pi b v^{2}}\left(8 b v^{2}-E^{2}\right)^{1 / 2}, & |E| \leq R_{0}=v \sqrt{8 b}, \\
0, & |E|>R_{0} .
\end{array}\right.
$$

Here the free parameter $v$ is defined by the variance of the off-diagonal elements, $v^{2}=\left\langle H_{n m}^{2}\right\rangle$, and is irrelevant to the statistical properties of the spectra. It also does not influence the structure of eigenstates. For $b=N / 2$ the above expression, Eq. (9), recovers the Wigner semicircle law which is well known within conventional RMT.

\section{Theory of the fidelity decay}

\subsection{Diffusive regime}

For a comparison we recapitulate the results known for chaotic systems, which are valid in the experiments for the high frequency limit. The fidelity is evaluated using a RMT modeling for $H_{0}$ and $V$ within Eq. (1). In the case of conventional RMT models, the fidelity was found $[8,10]$ to be

$$
\begin{aligned}
& F^{\lambda}(t) \simeq \mathrm{e}^{-\lambda^{2} \mathcal{C}(t)} \\
& \mathcal{C}(t) \equiv t^{2}+t-\int_{0}^{t} \mathrm{~d} \tau \int_{0}^{\tau} \mathrm{d} \tau^{\prime} b_{2}\left(\tau^{\prime}\right),
\end{aligned}
$$

where $b_{2}$ is the two-point form factor for the Gaussian orthogonal ensemble [16] given by

$$
\begin{aligned}
& b_{2}(\tau)=1-2 \tau+\tau \ln (2 \tau+1)+\Theta(\tau-1)[2(\tau-1) \\
& \quad-\tau \ln (2 \tau-1)],
\end{aligned}
$$

in which $\Theta(t)$ is the Heaviside function. Since in the diffusive regime $\Lambda \gg 1$, the form factor $b_{2}$ can be approximated nicely by RMT predictions, we expect that Eq. (10) will describe the temporal behavior of fidelity in the diffusive regime. For small perturbations $\lambda \ll 1$, this can be approximated as a Gaussian

$$
F^{\lambda}(t) \sim \exp \left(-(\lambda t)^{2}\right) .
$$

At larger perturbations and for times $t<t_{\mathrm{H}}$, the decay is exponential,

$$
F^{\lambda}(t) \sim \exp \left(-\lambda^{2} t\right)
$$

\subsection{Localized regime}

Now we investigate the behavior of the fidelity in the localized regime $(\Lambda \ll 1)$. Here $H_{0}$ and $V$ are modeled with BRMs with a bandwidth $b \leq \sqrt{N}$. Let us define the following set of eigenvalues and eigenvectors associated with $H_{0}$ and $H_{\lambda}$ :

$$
H_{0}\left|n^{(0)}\right\rangle=E_{n}^{0}\left|n^{(0)}\right\rangle ; \quad H_{\lambda}\left|m^{\lambda}\right\rangle=E_{m}^{\lambda}\left|m^{\lambda}\right\rangle .
$$

The fidelity amplitude in Eq. (1) can then be rewritten as

$$
f^{\lambda}(t)=\sum_{n, m, k} c_{n}^{*} c_{k} T_{m k}^{*} T_{n m} \exp \left(\mathrm{i} \omega_{m n} t\right)
$$

where the kernel $T_{n m}=\left\langle n^{(0)} \mid m^{\lambda}\right\rangle$ is the amplitude of the so-called local density of states (LDoS) and $\omega_{m n}=$ $\left(E_{m}^{\lambda}-E_{n}^{(0)}\right) / \hbar$. The initial state expansion coefficient in the unperturbed basis is given by $c_{k}=\left\langle\psi_{0} \mid k^{(0)}\right\rangle$. Taking a disorder ensemble average yields

$$
\begin{gathered}
\left\langle f^{\lambda}(t)\right\rangle=\left\langle\sum_{n, m, k} c_{n}^{*} c_{k} T_{m k}^{*} T_{n m} \exp \left(\mathrm{i} \omega_{m n} t\right)\right\rangle \\
\approx \sum_{n, m, k}\left\langle c_{n}^{*} c_{k}\right\rangle\left\langle T_{m k}^{*} T_{n m}\right\rangle\left\langle\exp \left(\mathrm{i} \omega_{m n} t\right)\right\rangle,
\end{gathered}
$$

in which the above distributive property of the average comes from a RMT conjecture that eigenstates and eigenenergies are statistically independent. Firstly, the expansion coefficients are

$$
\begin{gathered}
c_{n}^{*} c_{k}=\left\langle\psi_{0} \mid n^{(0)}\right\rangle\left\langle k^{(0)} \mid \psi_{0}\right\rangle=\left(\sum_{i} \psi_{0, i} n_{i}^{(0)}\right) \\
\times\left(\sum_{j} \psi_{0, j} k_{j}^{(0)}\right)=\sum_{i, j} \psi_{0, i} \psi_{0, j} n_{i}^{(0)} k_{j}^{(0)} .
\end{gathered}
$$

Under the ensemble average, we get

$$
\left\langle c_{n}^{*} c_{k}\right\rangle \approx \sum_{i, j}\left\langle\psi_{0, i} \psi_{0, j}\right\rangle\left\langle n_{i}^{(0)} k_{j}^{(0)}\right\rangle
$$

where $\psi_{0, i}$ is the $i$-th component of the initial wave function in a Wannier basis, and $n_{j}^{(0)}$ is the $j$-th component of the $n$-th eigenstate of $H_{0}$, in the Wannier basis. Assuming that the eigenstates of $H_{0}$ are exponentially localized (Anderson localization), the average overlap between the localized states is fairly negligible, unless the two eigenstates are either (a) the same eigenstate $(i=j)$, or (b) have their localized peak within the localization length of another eigenstate. This yields for the second average

$$
\left\langle n_{i}^{(0)} k_{j}^{(0)}\right\rangle=\delta_{n, k} \delta_{i, j} l_{\infty}^{-1} \delta\left(i \leq l_{\infty}\right) .
$$

Placing this back into the expansion coefficient average contracts the sum as

$$
\begin{aligned}
& \left\langle c_{n}^{*} c_{k}\right\rangle \approx \sum_{i, j}\left\langle\psi_{0, i} \psi_{0, j}\right\rangle \delta_{n, k} \delta_{i, j} l_{\infty}^{-1} \delta\left(i \leq l_{\infty}\right) \\
& \approx \frac{\delta_{n, k}}{l_{\infty}} \sum_{j \leq l_{\infty}}\left|\psi_{0, j}\right|^{2} \approx \sigma \delta_{n, k},
\end{aligned}
$$

where in the last step we use the notation $\sigma=$ $l_{\infty}^{-1} \sum_{j \leq l_{\infty}}\left|\psi_{0, j}\right|^{2}$. We substitute this result back into the average fidelity amplitude, to eventually get

$$
\left\langle f^{\lambda}(t)\right\rangle \approx \sigma \sum_{n, m, k} \delta_{n, k}\left\langle T_{m k}^{*} T_{n m} \exp \left(\mathrm{i} \omega_{m n} t\right)\right\rangle
$$




$$
s \approx \sigma \sum_{n, m}\left\langle P_{n m}\right\rangle\left\langle\exp \left(\mathrm{i} \omega_{m n} t\right)\right\rangle
$$

where $P_{n m}=\left|T_{m n}\right|^{2}$ is the LDoS kernel. The fidelity amplitude then reads

$$
\left\langle f^{\lambda}(t)\right\rangle \approx \sum_{n, m}\left\langle P_{n m}\right\rangle \exp \left(\mathrm{i}\left\langle\omega_{m n}\right\rangle t\right) .
$$

We shall now evaluate Eq. (22) for two different perturbation regimes.

\subsubsection{Localized regime with small perturbation}

For small perturbations $(\lambda \ll \Delta / \sigma)$, the LDoS kernel ought to be delta-like; the only levels that mix are separated within the mean level spacing. Numerically, we calculate the LDoS kernel for $\Lambda=0.1, \lambda=0.001$, and compare to its first-order perturbative approximation (FOPT) [27-29], which reads

$$
P_{n m} \approx \begin{cases}1, & n=m, \\ \frac{\lambda^{2}\left|V_{n m}\right|^{2}}{\left|E_{n}^{(0)}-E_{m}^{(0)}\right|^{2}}, & n \neq m .\end{cases}
$$

This comparison is shown in Fig. 4. The numeric LDoS

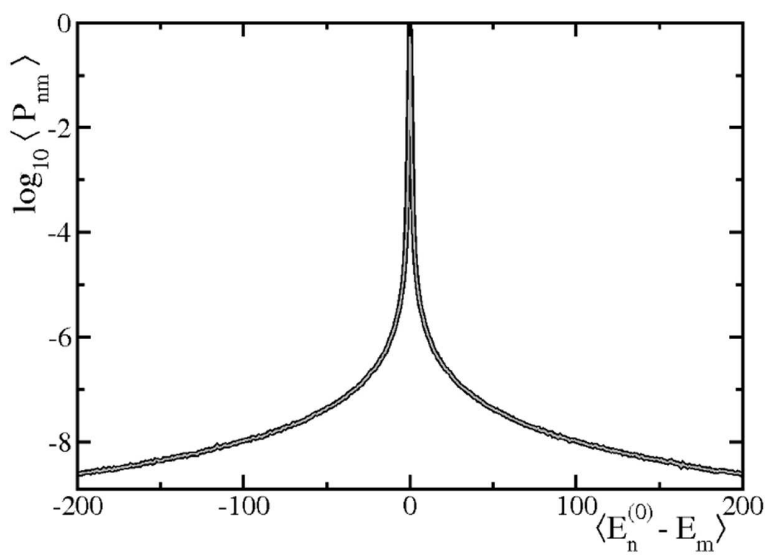

Fig. 4. LDoS kernel $P_{n m}$ for $\Lambda=0.1, \lambda=0.001$ (standard perturbative regime). The black line is the numerical LDoS kernel, while the grey line is the FOPT approximation. The mean level spacing is $\Delta \approx 1$.

is shown in black, and the FOPT approximation is shown in grey - a good match is seen between the two. The mean level spacing is $\Delta \approx 1$. For such small perturbations, numerics thus follow the expectation that the LDoS is delta-like. Approximating the LDoS kernel by a delta function then contracts across $m$ in Eq. (22) to yield

$$
\begin{aligned}
& f(\tau=t / \hbar) \approx \sum_{n}\left\langle\exp \left(\mathrm{i}\left(E_{n}^{\lambda}-E_{n}^{(0)}\right) \tau\right)\right\rangle \\
& \quad \approx \sum_{n} \exp \left(\mathrm{i} \lambda \nu_{n} \tau\right),
\end{aligned}
$$

in which we have used $\nu_{n}=\left(E_{n}^{\lambda}-E_{n}^{(0)}\right) / \lambda$, the definition of the level velocity. Turning the sum into a weighted integral over level velocities one gets

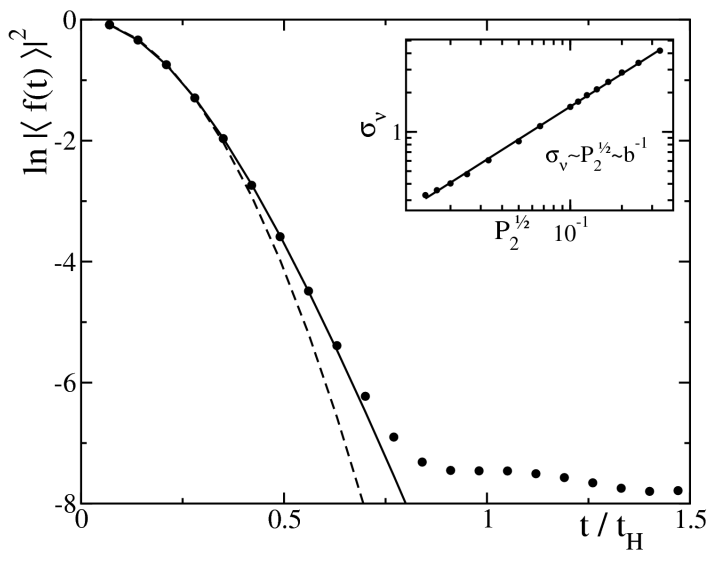

Fig. 5. The numerical fidelity from Eq. (15) for $\lambda=$ 0.001 (standard perturbative regime) for the localization parameter $\Lambda=0.0018 \ll 1$. The numerical results are the dots, the solid line is a best fit to Eq. (27), and the dashed line is the best fit to Eq. (10). A better fit is observed for Eq. (27). Inset: The variance parameter $\sigma_{\nu}$ is extracted from the fitting parameter $\alpha$ for different localization parameters, and plotted against $\sqrt{P_{2}}$, calculated directly from the eigenstates. The linear relation $\sigma_{\nu} \sim \sqrt{P_{2}}$ for localized eigenstates is verified, as the straight line is a best linear fit.

$$
f(\tilde{\tau}=\lambda \tau) \approx \int_{0}^{\infty} P(\nu) \mathrm{d} \nu \exp (\mathrm{i} \nu \tilde{\tau}),
$$

in which the fidelity is the Fourier transform over the level velocity probability distribution function (LVPDF), $P(\nu)$. The LVPDF has been studied thoroughly using both RMT [30] and field-theoretical techniques [31]. In the case of a BRM the LVPDF was found [30] to be

$$
P(\eta)=\frac{\pi}{6} \frac{\operatorname{coth}\left[\frac{\pi \eta}{\sqrt{6}}\right]-\sqrt{6}}{\sinh ^{2}\left[\frac{\pi \eta}{\sqrt{6}}\right]},
$$

where $\eta=\nu / \sigma_{\nu}$ is the variance-rescaled level velocity. Furthermore, it was shown [30] that the variance of the level velocity within the localized limit is equivalent to the root of the IPN and the bandwidth, $\sigma_{\nu}=\sqrt{P_{2}}=b^{-1}$. Taking the Fourier transform of Eq. (26) then yields the following result for the fidelity amplitude, in which all rescaling variables are contained in $\alpha$ :

$$
f(t)=(\alpha t)^{2} \operatorname{csch}(\alpha t), \quad \alpha=\lambda \sqrt{1.5 P_{2}} .
$$

This has to be seen in contrast to Eq. (12) for the diffusive regime.

To verify these analytical results, we calculate the fidelity numerically, and fit to two Eqs. (10) and (27). The results for a typical localization parameter $\Lambda \ll 1$ is shown in Fig. 5. It is seen that the novel decay of Eq. (27) better fits the numerical data than the full band (GOE) Gaussian decay of Eq. (10). For multiple such fits, the fitting parameter $\alpha$ is extracted, and compared to the $\sqrt{P_{2}}$ found directly from the eigenstates of $H_{\lambda}$. Within the inset, we verify the $\sqrt{P_{2}}$-dependence of the fit parameter $\alpha$, suggesting Eq. (27) as the model to use 
for the fidelity of localized modes in the standard perturbative regime.

\subsubsection{Localized regime with moderate perturbation}

Now we investigate the Wigner Lorentzian regime, where the perturbation strength $\lambda$ is moderate and energy levels mix beyond the mean level spacing, i.e. $\Delta / \sigma<$ $\lambda<\sqrt{b} \Delta / \sigma$. Infinite order perturbation theory yields a LDoS kernel of the form [28]:

$$
P_{n m} \approx \frac{\lambda^{2}\left|V_{n m}\right|^{2}}{\gamma^{2}+\left|E_{n}^{(0)}-E_{m}^{(0)}\right|^{2}}
$$

consisting of a non-perturbative core region of width $\gamma$ greater than the mean level spacing, $\gamma \gg \Delta$. In Fig. 6 we present a numerical result for the LDoS kernel corresponding to the BRM model with the localization parameter $\Lambda=0.1$ and for a value of the perturbation parameter $\lambda=0.1$. A nice agreement with a Lorentzian form of Eq. (28) can be seen. We next substitute a Lorentzian

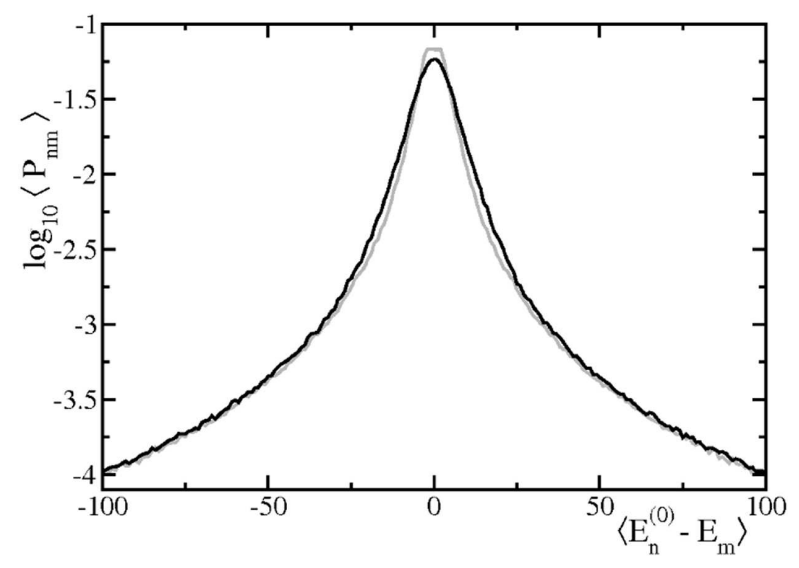

Fig. 6. LDoS kernel $P_{n m}$ for $\Lambda=0.1, \lambda=0.1$ (Wigner regime). The black line is the numerical LDoS kernel, while the grey line is the theoretical result of Eq. (28). Both are Lorentzian curves of width $\gamma$, found from normalization of the curves. The mean level spacing is $\Delta \approx 1$.

LDoS kernel within the localized mode result of Eq. (22) which in turn, gives for the fidelity

$$
\left\langle f^{\lambda}\left(\tilde{\tau}=t / t_{\mathrm{H}}\right)\right\rangle \sim \exp (-\gamma \tilde{\tau}),
$$

where $\tilde{\tau}=\Delta t / \hbar=t / t_{\mathrm{H}}$. Therefore within the Wigner regime, fidelity of localized modes is expected to display exponential decays similar to those seen for diffusive modes (see Eq. (13)). As a verification of this, the Lorentzian width should scale as $\gamma \sim \lambda^{2}$. In Fig. 7, we report (see solid line) our simulations for the fidelity decay. The dashed line in the main portion of Fig. 7 is a best fit of the numerical fidelity to the exponential result of Eq. (29). Within the inset, we numerically verify the Lorentzian width scales as $\gamma \sim \lambda^{2}$ for localized modes, as shown by the dash-dotted line. The single dot corresponds to the perturbation strength used in the main figure.

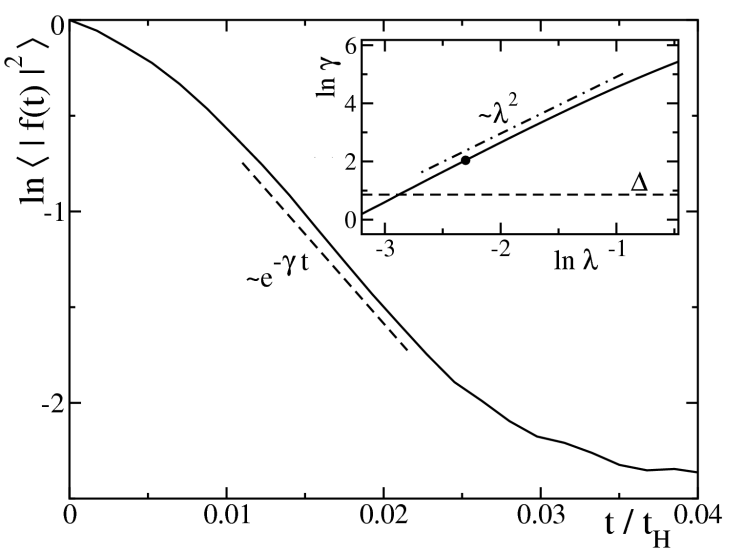

Fig. 7. The numerical fidelity from Eq. (15) for $\lambda=0.1$ (Wigner regime) is plotted for a localization parameter of $\Lambda=0.1$ (solid line). The dashed line is a best fit to Eq. (29). The fit is offset slightly to the left of its range for clarity. Within the inset, we numerically verify the relation $\gamma \sim \lambda^{2}$ for the Wigner regime at localization. The dashed line shows the boundary between standard perturbative and Wigner regimes, at which point $\gamma \sim \Delta$. The dash-dotted line shows the $\gamma \sim \lambda^{2}$ dependence. The single dot shows the value of $\gamma$ for the decay seen in the main figure.

\section{Experimental results}

\subsection{Diffusive regime}

Perturbations are geometric in nature (changing cavity length), so we can geometrically construct the matrix element of the perturbation as $[27,32,10,33]$

$$
\left(H_{1}\right)_{n m}=w \int_{0}^{L} \mathrm{~d} y \nabla_{\perp} \psi_{n}(y) \cdot \nabla_{\perp} \psi_{m}(y) .
$$

Since $\lambda^{2}=\left\langle\left(H_{1}\right)_{n m}^{2}\right\rangle$, we can now use a Berry conjecture of random plane wave superposition. Close to a straight boundary wall with Dirichlet conditions, the correlations that result in taking an average over the above matrix are Bessel in nature. Integration of the Bessel [10] correlations yields

$$
\lambda^{2}=\left\langle\left(H_{1}\right)_{n m}^{2}\right\rangle=\frac{4 k^{2} w^{2} L}{A^{2}} \frac{8}{3 \pi} .
$$

Wave function correlations approximated as the Bessel functions are ultimately semiclassical in nature, therefore the approximation for $\lambda$ above works very well for high wave numbers. For $\Delta \sim 1$, the area can be set to $A=4 \pi$ to yield

$$
\lambda^{2}=\frac{2 L}{3 \pi^{3}} k^{3} w^{2}=\frac{16 L}{3 c^{3}} \nu^{3} w^{2} .
$$

It is important to note then that from the above result, we have an expected scaling relation of $\lambda \sim w$ for chaotic cavities [10]. Within the diffusive frequency window $(10.5-12.0 \mathrm{GHz})$ the same relation holds, i.e. $\lambda \sim w$. In performing the calculation, we look at the fidelity resulting from the bulk antenna reflections, $S_{22}(E)$. The main purpose for this rests in the simple fact that the 
other antenna is too close to the shifting wall - the perturbation appears locally to this antenna - therefore the strength is much stronger, resulting in an extremely fast fidelity decay for $S_{11}(E)$. In Fig. 8, we present one such respective scattering fidelity decay, for $S_{22}(E)$. The

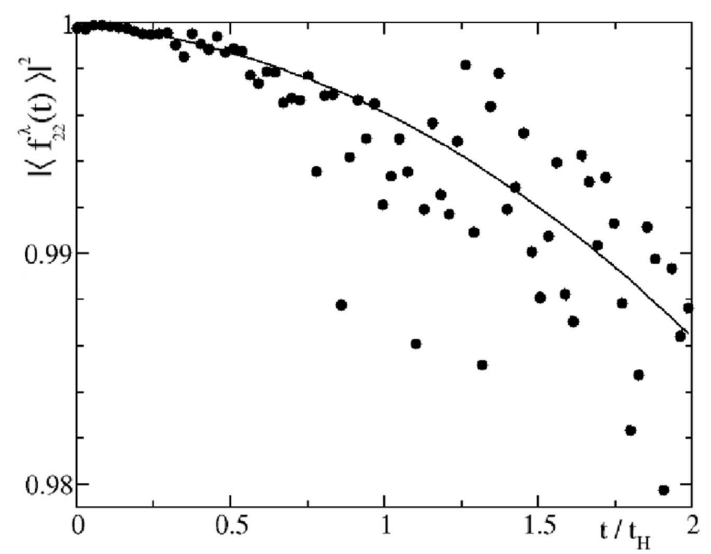

Fig. 8. A typical fidelity decay in the diffusive frequency window of $10.5-12 \mathrm{GHz}$. The dimensionless wallshift is $w / \delta w=2$. The solid line is a best fit of the data to Eq. (10). The resulting fit parameter $\lambda$ is compared against $w / \delta w$ in Fig. 9 in order to verify the scaling of Eq. (32).

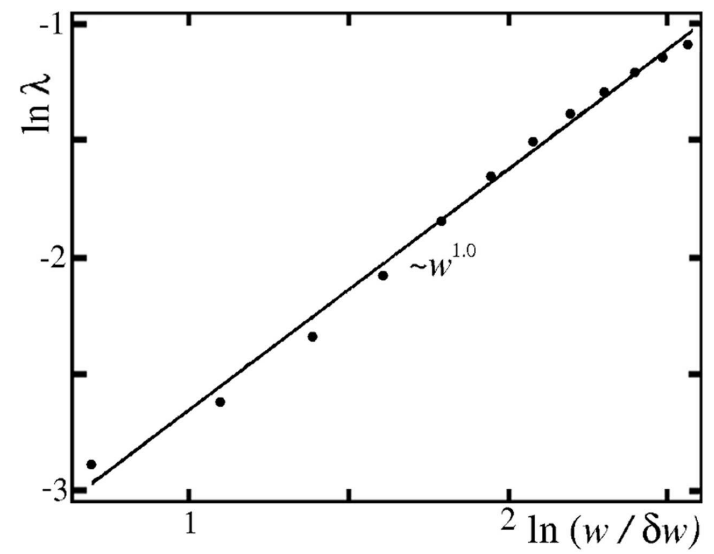

Fig. 9. Within the diffusive frequency window, the scaling of the free-fit parameter $\lambda$ of Eq. (10) with the dimensionless wallshift $w / \delta w$. The dots represent the extracted data. The solid line is the best fit to a power law relation $\lambda \sim w^{\eta}$. A power of $\eta=1.0 \pm 0.05$ is found, confirming the validity of Eq. (10).

points represent the actual scattering fidelity, while the solid line is the best fit of the numerical data to the traditional RMT behavior of Eq. (10), where $\lambda$ is the fitting parameter. All fitting was done in the standard perturbative regime for $t<t_{\mathrm{H}}$. We then perform this fitting procedure for a variety of wallshifts $w$, with the end purpose to compare the free fitting parameter $\lambda$ to the wallshift $w$. This is seen in Fig. 9, in which a scaling relation of $\lambda \sim w^{\eta}$ is shown. A power law of $\eta \sim 1.0 \pm 0.05$ is found, confirming our expectation for diffusive cavities, namely $\lambda \sim w$. It is very important to note that the scaling of the free-fit parameters with the wallshift is what verifies whether the outcome of RMT, Eq.(10), for the fidelity decay is applicable in the diffusive frequency window.

\subsection{Localized regime with small perturbation}

Within the standard perturbative regime, the theoretical fidelity behavior in the localized frequency window $(6.0-7.5 \mathrm{GHz})$ is the novel given by Eq. (27). Within this equation, we will treat $\alpha \sim \sqrt{P_{2}} w$ as the free-fitting parameter, to give a scaling expectation of $\alpha \sim w$. In addition to fitting the experimental data of scattering fidelity in the localized frequency window to our novel decay prediction of Eq. (27), we also fit the experimental decay to the traditional RMT decay of Eq. (10) with $\lambda$ as a free-fit parameter; in a similar fashion to what was done in the previous theoretical/numerical section (see Fig. 5). A representative experimental fidelity curve and the fits are shown in Fig. 10. The data points represent experimental data, the solid line is the best fit to the novel decay behavior of Eq. (27) while the dashed line is the best fit to the traditional RMT decay of Eq. (10).

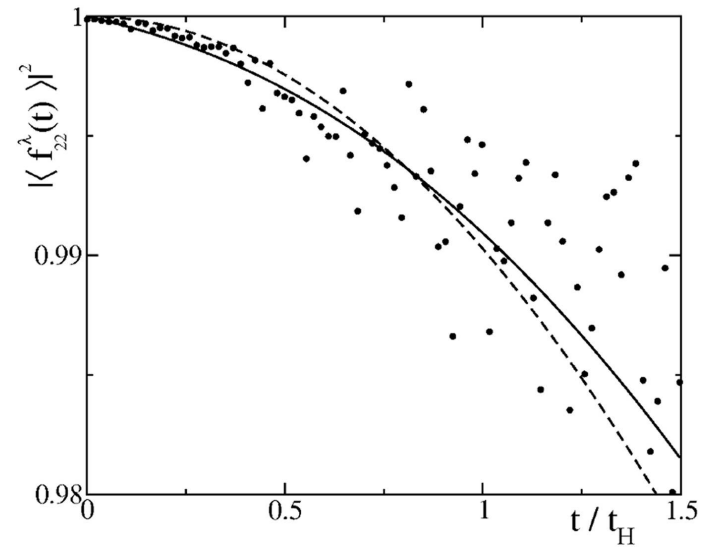

Fig. 10. A typical fidelity decay for the standard perturbative regime in a localized frequency window. The points are the experimental data. The solid line represents a best fit to the novel decay behavior of Eq. (27), while the dashed line represents a best fit to the traditional RMT decay behavior of Eq. (10). A better fit to the novel behavior of Eq. (27) is observed.

The result is seen by eye - within the localized frequency window, the novel decay of Eq. (27) fits better than the traditional RMT decay of Eq. (10). This can further be quantified in the scaling of the free-fit parameters. Whichever free-fit parameter ( $\alpha$ for the novel decay and $\lambda$ for the traditional RMT decay) best scales as $\sim w$ ultimately tells which model performs best. The scaling analysis is performed in Fig. 11, in which the free-fit parameters are plotted against the dimensionless wallshift, $w / \delta w$, and then fit to a power law of $\alpha, \lambda \sim w^{\eta}$. Within the figure the squares correspond to $\alpha$ and the dots correspond to $\lambda$. For the novel behavior of Eq. (27), a scaling 


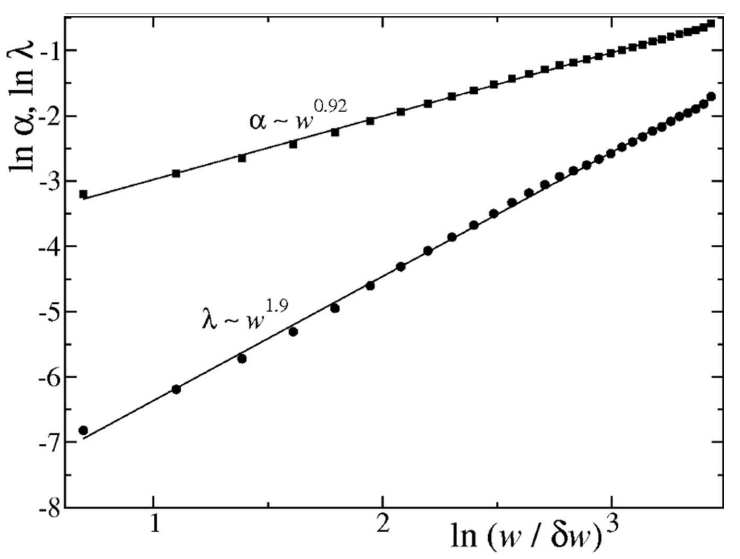

Fig. 11. Scaling analysis of the fitting parameters within the localized frequency window. The squares denote the $\alpha$ parameter from Eq. (27) while the dots denote the $\lambda$ parameter from Eq. (10). The straight lines indicate a power law fit to $\alpha, \lambda \sim w^{\eta}$. The $\alpha$ then scale properly as $\eta=0.92 \pm 0.05$, suggesting Eq. (27) as the correct fit. The failure of the $\lambda$ parameter to scale as $\lambda \sim w$ (scales as $\eta=1.9 \pm 0.05$ ) additionally shows Eq. (27) as the better description of fidelity decay in localized scattering systems.

of $\eta=0.92 \pm 0.05$ was obtained. For the case of a fit to traditional RMT, Eq. (10), a scaling of $\eta=1.9 \pm 0.05$ was obtained. Since the novel behavior gave a better scaling $(\eta \sim 1)$, it is indeed the proper model to use within the localized frequency window. To close this regime, an emphasis on the relation $\alpha \sim w \sqrt{P_{2}}$ cannot be overstated; this relation dictates the slope of the straight line in Fig. 11, and may therefore be used to extract out the inverse participation number in the event of a localized mode.

\subsection{Localized regime with moderate perturbation}

Within the Wigner regime, the fidelity for the localized frequency window was found to have a theoretical expectation of Eq. (29), similar to the decay in chaotic/diffusive systems. The crossover between the standard perturbative and Wigner regimes was observed by eye where the fidelity decay of Eq. (27) began failing to capture the decay behavior, typically occurring at wallshifts with the chaotic perturbation strength, given by Eq. (32), of $\lambda \approx 1$. The decay rate of $\gamma$ was extracted by fitting the region $0.3<t / t_{\mathrm{H}}<1$ to the exponential decay of Eq. (29). A typical fidelity decay in this regime is shown in Fig. 12, in which the points are the experimental data. The solid line is the exponential fit of Eq. (29) within the aforementioned region. The fitting is performed for several different wallshifts, and the free-fit parameter $\gamma$ is then plotted against the dimensionless wallshift $(w / \delta w)$ in Fig. 13. A power law scaling of $\gamma \sim w^{\eta}$ is then extracted, and a power of $\eta=2.3 \pm 0.05$ is found. As opposed to $\alpha, \lambda$ in the standard perturbative regime, the theoretical scaling expectation in the Wigner

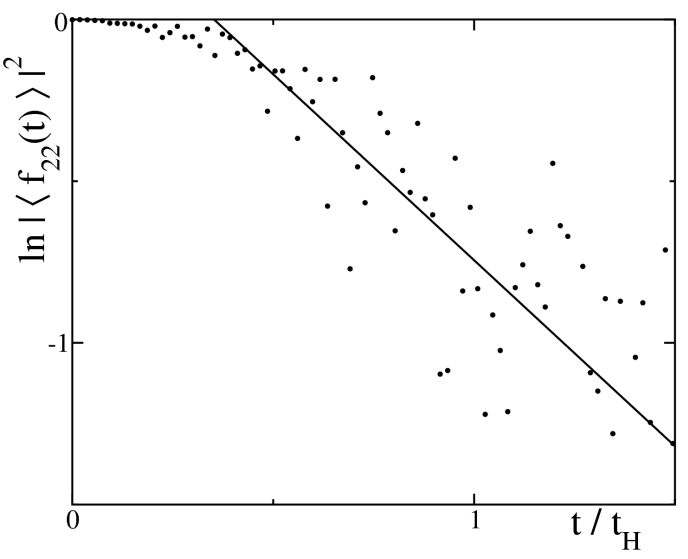

Fig. 12. A typical fidelity decay for the Wigner regime in a localized frequency window. The points are the experimental data. The solid line represents a best fit of the region $0.3<t / t_{\mathrm{H}}<1$ to the exponential decay of Eq. (29), which captures the decay behavior nicely.

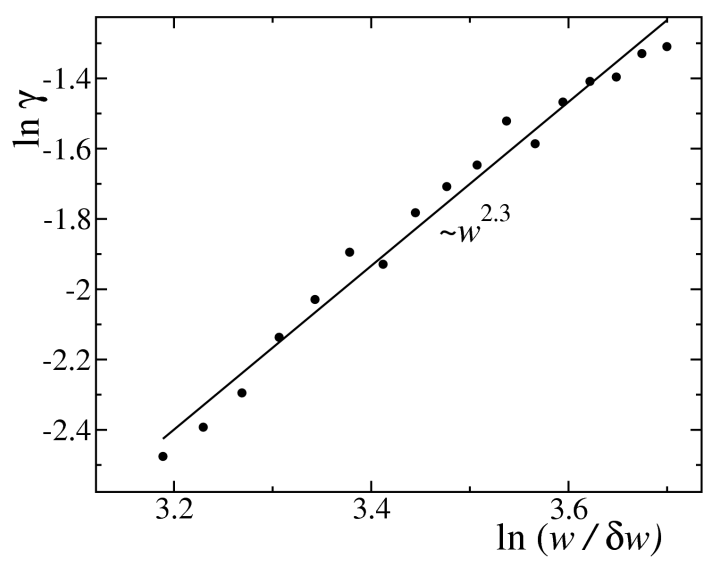

Fig. 13. Scaling analysis of the exponential fitting parameter $\gamma$ from Eq. (29), for localized fidelity in the Wigner regime. The dots denote the $\gamma$ parameter. The straight line indicates a power law fit to $\gamma \sim w^{\eta}$, with a power of $\eta=2.3 \pm 0.05$. The experimental data then captures the correct scaling of $\sim w^{2}$.

regime was found to be $\gamma \sim w^{2}$. Therefore, we also capture the correct decay behavior within the localized frequency window for the Wigner regime.

\section{Conclusion}

In conclusion, using echo dynamics, we were able to isolate absorption phenomena and identify unambiguously traces of localization in random media. Our theoretical calculations, based on an RMT modeling, indicated that the inverse participation ratio of localized modes dictates the fidelity behavior. Therefore a novel decay law in the standard perturbative regime is predicted. The novel decay could be verified experimentally and it can be used as a reliable to discriminate between localized and diffusive regimes in the presence of absorption. Stronger 
perturbations within the Wigner regime see the fidelity of localized modes behaving in an exponential decay similar to diffusive modes.

\section{Acknowledgments}

The research was funded by the Deutsche Forschegruppe 760 and by the US-Israel Binational Science Foundation (BSF), Jerusalem, Israel. Computational time at Wesleyan was provided by NSF grant No. CNS-0619508.

\section{References}

[1] P. Anderson, Phys. Rev. 109, 1492 (1958).

[2] M.E. Gertsenshtein, V.B. Vasil'ev, Teor. Veroyatn. Prim. 4, 391 (1959); 5, 240(E) (1960).

[3] S. John, Phys. Rev. Lett. 53, 2169 (1984).

[4] T. Schwartz, G. Bartal, S. Fishman, M. Segev, Nature 446, 05623 (2007); H. Hu, A. Strybulevych, J.H. Page, S.E. Skipetrov, B.A. van Tiggelen, Nature-Phys. 4, 945 (2008); J. Billy, V. Josse, Z. Zuo, A. Bernard, B. Hambrecht, P. Lugan, D. Clément, L. Sanchez-Palencia, P. Bouyer, A. Aspect, Nature 453, 891 (2008).

[5] H. De Raedt, A. Lagendijk, P. de Vries, Phys. Rev. Lett. 62, 47 (1989).

[6] A. Chabanov, M. Stoytchev, A. Genack, Nature 404 850 (2000); A. Chabanov, A. Genack, Phys. Rev. Lett. 87, 233903 (2001); A. Genack, A. Chabanov, J. Phys. A 38, 10465 (2005).

[7] J.T. Edwards, D.J. Thouless, J. Phys. Chem. 5, 807 (1972).

[8] T. Gorin, T. Prosen, T.H. Seligman, M. Znidarič, Phys. Rep. 435, 33 (2006); Ph. Jacquod, C. Petitjean, Adv. Phys. 58, 67 (2009)

[9] S.A. Gardiner, J.I. Cirac, P. Zoller, Phys. Rev. Lett. 79, 4790 (1997); M.F. Andersen, A. Kaplan, N. Davidson, Phys. Rev. A 64, 043801 (2001); S. Kuhr, W. Alt, D. Schrader, I. Dotsenko, Y. Miroshnychenko, W. Rosenfeld, M. Khudaverdyan, V. Gomer, A. Rauschenbeutel, D. Meschede, Phys. Rev. Lett. 91, 213002 (2003).

[10] R. Schäfer, T. Gorin, T.H. Seligman, H.-J. Stöckmann, New J. Phys. 7, 152 (2005); Phys. Rev. Lett. 95, $184102(2005)$.

[11] O.I. Lobkis, R.L. Weaver, Phys. Rev. Lett. 90, 254302 (2003).

[12] M.A. Nielsen, I.L. Chuang, Quantum Computation and Quantum Information, Cambridge Uni. Press, Cambridge 2000

[13] R.A. Jalabert, H.M. Pastawski, Phys. Rev. Lett. 86, 2490 (2001)

[14] Ph. Jacquod, P.G. Silvestrov, C.W.J. Beenakker, Phys. Rev. E 64, 055203 (2001).
[15] N.R. Cerruti, S. Tomsovic, Phys. Rev. Lett. $\mathbf{8 8}$ 054103 (2002); J. Phys. A 36, 3451 (2003).

[16] T. Gorin, T. Prosen, T.H. Seligman, New J. Phys. 6, 1 (2004); T. Prosen, M. Žnidarič, J. Phys. A 35, 1455 (2002).

[17] G. Benenti, G. Casati, Phys. Rev. E 66, 066205 (2002).

[18] Y. Adamov, I.V. Gornyi, A.D. Mirlin, Phys. Rev. E 67, 056217 (2003).

[19] T. Kottos, D. Cohen, Europhys. Lett. 61, 431 (2003); M. Hiller, T. Kottos, D. Cohen, T. Geisel, Phys Rev. Lett. 92, 010402 (2004); G.S. Ng, J. Bodyfelt, T. Kottos, Phys. Rev. Lett. 97, 256404 (2006).

[20] R. Höhmann, U. Kuhl, H.-J. Stöckmann, Phys. Rev. Lett. 7, 152 (2005); Phys. Rev. Lett. 100, 124101 (2008).

[21] J.D. Bodyfelt, M.C. Zheng, T. Kottos, U. Kuhl, H.-J. Stöckmann, Phys. Rev. Lett. 102, 253901 (2009).

[22] H.U. Baranger, P.A. Mello, Phys. Rev. Lett. 73, 142 (1994).

[23] A.D. Mirlin, R. Pnini, B. Shapiro, Phys. Rev. E 57, R6285 (1998).

[24] K. Efetov, Supersymmetry in Disorder and Chaos, Cambridge Uni. Press, Cambridge 1997.

[25] Y.V. Fyodorov, A.D. Mirlin, Phys. Rev. Lett. 67, 2405 (1991); Y.V. Fyodorov, A.D. Mirlin, J. Phys. A 26, L551 (1993); Y.V. Fyodorov, A.D. Mirlin, Int. J. Mod. Phys. B 8, 3795 (1994).

[26] G. Casati, I. Guarneri, F. Izrailev, L. Molinari, K. Życzkowski, Phys. Rev. Lett. 72, 2697 (1994); F. Izrailev, Chaos, Solitons Fractals 5, 1219 (1995); T. Kottos, A. Politi, F.M. Izrailev, S. Ruffo, Phys. Rev. E 53, R5553 (1996); F.M. Izrailev, T. Kottos, A. Politi, G.P. Tsironis, Phys. Rev. E 55, 4951 (1997); T. Kottos, A. Politi, F.M. Izrailev, J. Phys. C 10, 5965 (1998); T. Kottos, F.M. Izrailev, A. Politi, Physica D 131, 155 (1999); A.J. Mendez-Bermudez, T. Kottos, Phys. Rev. B 72, 064108 (2005).

[27] D. Cohen, Ann. Phys. 283, 175 (2000).

[28] D. Cohen, T. Kottos, Phys. Rev. E 63, 36203 (2001); M. Hiller, T. Kottos, T. Geisel, Phys. Rev. A $\mathbf{7 3}$, 061604 (2006).

[29] M. Hiller, D. Cohen, T. Geisel, T. Kottos, Ann. Phys. 321, 1025 (2006)

[30] P. Kunstman, K. Życzkowski, J. Zakrzewski, Phys. Rev. E 55, 2446 (1997).

[31] Y.V. Fyodorov, Phys. Rev. Lett. 73, 2688 (1994).

[32] A. Barnett, D. Cohen, E.J. Heller, J. Phys. A 34, 413 (2001).

[33] H.-J. Stöckmann, Quantum Chaos: An Introduction, 1st ed., Cambridge University Press, Cambridge 1999. 